than culture or microscopy, but specificity at extra-genital sites may be lower due to cross reactivity with other Neisseria species. BASHH recommends supplementary testing of NG positive extragenital NAATs to improve specificity. This inner city DGH introduced supplementary testing on 01/11/13.

Aim(s)/objectives To evaluate the impact of introducing supplementary testing on the rate of extra-genital NG diagnosis and concordance of positive NAATs with culture.

Methods All patients with a diagnosis of NG at any site between 01/08/13 and 31/01/14 were identified. Concordance of positive NAATs with bacterial culture pre- and post-intervention was reviewed.

Results There were 471 positive NAATs from 372 patients during the study period. Extra-genital samples accounted for $48.6 \%(\mathrm{n}=118 / 243)$ of positive NAATS pre-intervention and $41.2 \%(\mathrm{n}=94 / 228)$ post-intervention, $(\mathrm{p}=0.03)$. Culture was obtained from 305 sites, 119 of which were extra-genital. Concordance pre- and post-intervention is detailed in below.

\begin{tabular}{llll}
\hline Abstract P19 & Table 1 & Gonorrhoea test concordance \\
\hline & \multicolumn{3}{c}{ Proportion of cultures positive for NG } \\
\cline { 2 - 4 } & Pre intervention & Post intervention & $P$ value \\
\hline Rectal & $9 / 21(42.8 \%)$ & $10 / 19(53.0 \%)$ & 0.39 \\
Pharyngeal & $3 / 49(6.10 \%)$ & $3 / 31(9.67 \%)$ & 0.40 \\
\hline
\end{tabular}

Discussion/conclusion The proportion of NG positive NAATs from extra-genital sites fell following the introduction of supplementary testing, which may reflect an improvement in specificity. A non-significant increase in concordance of NAATs with culture was noted however concordance was low overall, especially in the pharynx. It remains unclear whether discordant results represent lower sensitivity of culture, lower specificity NAATs despite supplementary testing, or spontaneous clearance between screening and recall for culture.

\section{P20 INVESTIGATION INTO AN INCREASE OF DIAGNOSES OF GONORRHOEA IN SOUTHEND-ON-SEA}

${ }^{1}$ Lynsey Emmett* ${ }^{2}$ Henna Jaleel, ${ }^{2}$ Mohd Sabri Abu Bakar, ${ }^{2}$ Donna Stookes, ${ }^{3}$ Karen Payne, ${ }^{4}$ Simon Ford, 'Mark Reacher, 'Sultan Salimee. 'Public Health England, East, UK; ${ }^{2}$ Southend University Hospital NHS Foundation Trust, Southend-on-Sea, UK; ${ }^{3}$ South Essex Partnership University NHS Foundation Trust, Southend-on-Sea, UK; ${ }^{4}$ Southend-onSea Borough Council, Southend-on-Sea, UK

\subsection{6/sextrans-2015-052126.64}

Background/introduction Between 2012 and 2013 the rate of gonorrhoea in Southend increased significantly from 24.6 to 42.4 per 100,000 . A multidisciplinary Incident Management Team was established in June 2014 to assess the situation and implement appropriate control measures. However, the number of cases had already begun to fall. A retrospective case review was initiated.

Aim(s)/objectives To identify factors that contributed to the increase and subsequent decrease in diagnoses.

Methods Enhanced questionnaires were completed for each case diagnosed between October 2012 and March 2014. Antibiotic resistance profiles were provided by the local laboratory.
Previous STI and HIV test history was extracted from the Genitourinary Medicine Clinic Activity Dataset (GUMCADv2).

Results Provisional results show that enhanced forms were completed for 160 cases. Majority of cases were of white ethnicity $(64 \%)$ and born in the UK (87\%). Cases were aged between 15 to 63 years (median 28 years), 62\% were male and 60\% heterosexual. Most cases had 1 or 2 partners in the preceding 3 months and attended because of symptoms (40\%). However, approximately 30 cases had been referred from a level 2 service - some of which had negative results when re-tested. The majority of cases were treated with first line therapy and had a test of cure undertaken.

Discussion/conclusion Full details of the epidemiology, presentation and diagnosis of the cases will be presented - including a comparison with the cases diagnosed before and after the increase, the use of social network techniques and an analysis against the auditable outcome measures in the BASHH guidelines.

\section{P21 IS IT SYPHILIS? THE DARK ART OF INTERPRETING SYPHILIS SEROLOGY}

Hemanti Patel, Sarah Alexander, Helen Fifer*. Public Health England, London, UK

\subsection{6/sextrans-2015-052126.65}

Background/introduction The diagnosis of syphilis relies mainly upon a panel of serological tests. A sensitive treponemal test such as the enzyme immunoassay (EIA) is used as a screening test; another treponemal test such as the Treponema pallidum particle agglutination (TPPA) assay is used to confirm a reactive screening test. Difficulties arise when these tests produce a discordant result. Our laboratory uses the INNO-LIA immunoblot assay to resolve discordant screening results.

Aim(s)/objectives To evaluate whether the use of the INNO-LIA enables clinically useful interpretation.

Methods We reviewed the last 100 INNO-LIA tests performed by our laboratory.

Results Comparison of EIA, TPPA and INNO-LIA results are shown in Table 1. The Antibody Index is a measure of the positive signal in the EIA ( $\geq 1.2$ is a positive result).

Discussion/conclusion In EIA positive (AI $\geq 5$ )/TPPA equivocal cases the INNO-LIA was always positive or equivocal, consistent with treponemal infection. The INNO-LIA test may be unnecessary in these cases. In EIA positive/TPPA negative cases, the INNO-LIA is able to resolve the discordant result less than half of the time. Overall the INNO-LIA produced equivocal results in $44 \%$ of serums, which is unsatisfactory for confirming the diagnosis of syphilis. Although the INNO-LIA does help resolve some cases, there remains a need for new diagnostics.

\begin{tabular}{|c|c|c|c|c|c|c|c|}
\hline \multirow{2}{*}{$\begin{array}{l}\text { No. of } \\
\text { samples }\end{array}$} & \multirow{2}{*}{ EIA } & \multirow{2}{*}{$\begin{array}{l}\text { EIA } \\
\text { Antibody } \\
\text { Index (AI) }\end{array}$} & \multirow{2}{*}{ TPPA } & \multicolumn{3}{|c|}{ INNO-LIA } & \multirow{2}{*}{$\begin{array}{l}\text { Discordan } \\
\text { result } \\
\text { resolved? }\end{array}$} \\
\hline & & & & Negative & Positive & Equivocal & \\
\hline 41 & Positive & $\geq 5$ & Negative & 5 & 12 & 24 & $41 \%$ \\
\hline 30 & Positive & $\geq 5$ & Equivocal & 0 & 20 & 10 & $100 \%$ \\
\hline 26 & Positive & $\leq 5$ & Equivocal & 5 & 13 & 8 & $81 \%$ \\
\hline 3 & Positive & $\leq 5$ & Negative & 1 & 0 & 2 & $33 \%$ \\
\hline
\end{tabular}

